$\mathrm{PM} / 98-18$

GDR-S-013

\title{
IMPACT OF THE SUSY DECAYS ON THE SEARCH FOR THE MSSM HIGGS BOSONS AT THE LHC
}

\author{
A. DJOUADI \\ Laboratoire de Physique Mathématique et Théorique, UMR-CNRS 5825 \\ Université Montpellier II, F-34095 Montpellier Cedex 5, France.
}

\begin{abstract}
In the context of the Minimal Supersymmetric extension of the Standard Model, we discuss the impact of the decays of the neutral Higgs bosons into supersymmetric particles, charginos/neutralinos and sfermions. We show that these decay modes could be dominant, when they are kinematically accessible, thus strongly suppressing the branching ratios for the decay channels which are used to detect the Higgs bosons at hadron colliders. These SUSY decay modes should therefore not be overlooked in the search for the Higgs particles at the LHC.
\end{abstract}

\section{Introduction}

The search for Higgs bosons is one of the main entries in the LHC agenda. While the search for the Standard Model (SM) Higgs bosons has been shown to be rather straightforward, provided that a high luminosity $\int \mathcal{L} \sim 300 \mathrm{fb}^{-1}$ is collected by the ATLAS and CMS collaborations, the search for the Higgs particles of supersymmetric extensions (SUSY) of the SM, seems to be slightly more involved.2 3 The simplest version, the Minimal Supersymmetric Standard Model (MSSM), leads to the existence of five physical states: two $\mathrm{CP}$-even Higgs bosons $h$ and $H$, a $\mathrm{CP}$-odd Higgs boson $A$ and two charged Higgs particles $H^{ \pm}$In principle, many of the numerous decay modes and production processes of the MSSM Higgs bosons are needed to cover the full MSSM parameter space; see Ref.⿴囗十

However, in many scenarii such as mSUGRA, 3 the MSSM Higgs sector is in the so called decoupling regime for most of the SUSY parameter space allowed by present data constraints. The heavier $H, A$ and $H^{ \pm}$states are rather heavy and degenerate in mass, while the lightest $h$ boson reaches its maximal allowed mass value $M_{h} \lesssim 80-130 \mathrm{GeV} 6$ and has almost the same properties as the SM Higgs boson. In a large part of the MSSM parameter space, only the lightest $h$ boson can be produced at the LHC and, with some luck the heavy $H, A$ particles.

At the LHC, the most promising channel2 3 for detecting the lightest $h$ boson is the rare decay into two photons, $h \rightarrow \gamma \gamma$, with the Higgs particle dominantly produced via the top quark loop mediated gluon-gluon fusion mechanism $g g \rightarrow h^{a}$.

\footnotetext{
${ }^{a}$ Two other channels can also he used to detect the $h$ particle in this mass range: the production
} in association with a $W$ boson 
In the decoupling regime, the two LHC collaborations expect to detect the narrow $\gamma \gamma$ peak in the entire Higgs mass range, ${ }^{b} 80 \lesssim M_{h} \lesssim 130 \mathrm{GeV}$, with an integrated luminosity $\int \mathcal{L} \sim 300 \mathrm{fb}^{-1}$ corresponding to three years of LHC running.

The heavy $\mathrm{CP}$-even $H$ and $\mathrm{CP}$-odd $A$ bosons can be searched for at the LHC through their decays modes into $\tau^{+} \tau^{-}$pairs with the Higgs bosons produced in the $g g$ fusion mechanism or in association with $b \bar{b}$ pairs: $g g \rightarrow H / A$ and $g g, q \bar{q} \rightarrow$ $b \bar{b}+H / A$. 6 This needs large values $\tan \beta \gtrsim 5$ for masses $M_{H, A} \gtrsim 300 \mathrm{GeV}$, to enhance the production cross sections and the $\tau^{+} \tau^{-}$decay branching ratios, which in the case where only standard decay modes are allowed reach the asymptotic value of $\operatorname{BR}\left(h \rightarrow \tau^{+} \tau^{-}\right) \sim 10 \%$. The decays into muon pairs, $H / A \rightarrow \mu^{+} \mu^{-}$, give a rather clean signal and can be used despite of the very small branching ratios, which asymptotically reach the level of $\sim 4.10^{-4}$.

However, in these analyses, it is always assumed that the heavy $H / A$ bosons decay only into standard particles, and that the SUSY decay modes are shut. But for such large values of $M_{H, A}$, at least the decays into the lightest neutralinos and charginos, and possibly into to light $\tilde{t}$ and $\tilde{b}$, can be kinematically allowed. These modes could have large decays widths, and thus could suppress the $H / A \rightarrow \tau^{+} \tau^{-}$ branching ratios drastically. For the lightest $h$ boson, because of its small mass, only a little room is left for decays into SUSY particles by present experimental data However, the possibility of $h$ decays into neutralinos is not yet completely ruled out, especially if one relaxes the gaugino mass unification; decays into sneutrinos are also still possible. When these invisible decays occur, they can be dominant, hence reducing the probability of the $h \rightarrow \gamma \gamma$ decay to occur. These SUSY decays should therefore not be overlooked as they might jeopardize the detection of the Higgs particles at the LHC.

These SUSY decays of the Higgs bosons are discussed and updated in this note. All branching ratios are obtained with the help of an adapted version of the program HDECAY. 13 Previous analyses of SUSY Higgs boson decays can be found in Ref.14

\section{Invisible decays of the $h$ boson}

Despite the lower bound of $91 \mathrm{GeV}$ on the mass of the lightest chargino $\chi_{1}^{+}$and the constraints from $\chi_{0}^{1} \chi_{0}^{2}$ searches at LEP2 2 , the decay of the lightest $h$ boson into a pair of lightest neutralinos is still kinematically possible. Even in the constrained MSSM with a common gaugino mass at the GUT scale, leading to the well-know relation between the wino and bino masses $M_{1} \overline{\overline{1}} \frac{5}{3} \operatorname{tg}^{2} \theta_{W} M_{2} \sim \frac{1}{2} M_{2}$, the lower bound on the LSP mass is only $m_{\chi_{0}^{1}} \gtrsim 30 \mathrm{GeV} .5$ Since the upper bound on the lightest $h$ boson in the MSSM is $M_{h} \sim 130 \mathrm{GeV}$, there is still room for the invisible decay $h \rightarrow \chi_{0}^{1} \chi_{0}^{1}$ to occur.

\footnotetext{
${ }^{b}$ Note, however, that the rates in the $g g$ mechanism can be much smaller them expected if $\tilde{t}$ squarks are relatively light and their coupling to the $h$ boson strongly enhanced 10 Some compepsation however might come from the process $p p \rightarrow \tilde{t} \tilde{t} h$ which has rather large rates in thifocase.11

${ }^{c}$ For lower values $\tan \beta \lesssim 3$, most of which will be covered by the upgrade of LEP212 to $\sqrt{s}=200$ $\mathrm{GeV}$, and not too large $M_{H, A}$ vplues, the decays $H / A \rightarrow t \bar{t}, H \rightarrow h h \rightarrow b \bar{b} \gamma \gamma$ and $A \rightarrow Z h \rightarrow$ $l^{+} l^{-} b \bar{b}$ can also be used; see Ref.l.
} 
In the decoupling regime, the $h b \bar{b}$ coupling is SM-like and can be much smaller than the $h \chi_{1}^{0} \chi_{1}^{0}$ coupling; the decay of $h$ into the lightest neutralinos can be then dominant, resulting in a much smaller $\operatorname{BR}(h \rightarrow \gamma \gamma)$ than in the SM. Far from the decoupling limit, the coupling $g_{h b b} \sim \tan \beta$ is strongly enhanced for $\tan \beta \gtrsim 3$, while the $h$ boson couplings to $W$ bosons and top quarks [which provide the main contributions to the $h \gamma \gamma$ loop vertex] are suppressed. This again will result in a strong suppression of $\mathrm{BR}(h \rightarrow \gamma \gamma)$.

The partial width for the decay $h \rightarrow \chi_{0}^{1} \chi_{0}^{1}$ is given by

$$
\Gamma\left(h \rightarrow \chi_{0}^{1} \chi_{0}^{1}\right)=\frac{G_{F} M_{W}^{2} M_{h}}{2 \sqrt{2} \pi} g_{h \chi_{0}^{1} \chi_{0}^{1}}^{2} \beta_{\chi}^{3}
$$

with $\beta_{\chi}^{2}=1-4 m_{\chi_{1}^{0}}^{2} / M_{h}^{2}$ and the normalized coupling $g_{h \chi_{0}^{1} \chi_{0}^{1}}$ given by

$$
g_{h \chi_{1}^{0} \chi_{1}^{0}}=\left(Z_{12}-\tan \theta_{W} Z_{11}\right)\left(\sin \beta Z_{14}-\cos \beta Z_{13}\right)
$$

with $Z$ is the matrix diagonalizing the neutralino mass matrix. 0 The decay is important only for moderate values of $M_{2}$ and $\mu$ [with a preference for $\mu>0$ ] since the $h$ boson prefers to couple to neutralinos which are a mixture of gauginos and higgsinos. In this range, the decay $h \rightarrow \chi_{0}^{1} \chi_{0}^{1}$ is dominant if $M_{h}$ is above the $2 m_{\chi_{0}^{1}}$ threshold; close to this value, the width is strongly suppressed by the $\beta_{\chi}^{3}$ factor.

As an illustration of this possibility, we show in Fig. 1 the fraction $\operatorname{BR}(h \rightarrow \gamma \gamma)$ as a function of $\mu$ for two values of $\tan \beta=2,30$. We choose $M_{A}=m_{\tilde{q}}=1 \mathrm{TeV}$ and the "maximal mixing" scenario $A_{t}=\sqrt{6} m_{\tilde{q}}$ to maximize the $h$ boson mass [this gives $M_{h} \simeq 126 \mathrm{GeV}$ for $\tan \beta=30$ and $M_{h} \simeq 106 \mathrm{GeV}$ for $\tan \beta=2$; the variation with $\mu$ is almost negligible]. In the $\tan \beta=30$ and $M_{2}=140 \mathrm{GeV}$ case, for $|\mu| \gtrsim 200 \mathrm{GeV}$ the channel $h \rightarrow \chi_{1}^{0} \chi_{1}^{0}$ is kinematically closed and $\operatorname{BR}(h \rightarrow \gamma \gamma)$ is SM-like, $\simeq 2.3 \times 10^{-3}$. In the range $110 \lesssim|\mu| \lesssim 200 \mathrm{GeV}$, the LSP is lighter that $M_{h} / 2$ while the chargino is still heavier than $91 \mathrm{GeV}$, the decay $h \rightarrow \chi_{1}^{0} \chi_{1}^{0}$ is thus allowed to occur and suppresses $\operatorname{BR}(h \rightarrow \gamma \gamma)$. The suppression is stronger with decreasing $|\mu|$ since the phase-space becomes more favorable, and also the LSP tends to be an equal mixture of higgsino and gaugino. The maximum drop of $\operatorname{BR}(h \rightarrow \gamma \gamma)$ is a factor of three and two for $\mu>0$ and $\mu<0$ respectively. For values $|\mu| \lesssim 110 \mathrm{GeV}, m_{\chi_{1}^{ \pm}}$exceeds its experimentally allowed lower bound.

In the case $\tan \beta=2$ and $M_{2}=100 \mathrm{GeV}$, the only experimentally allowed region is $|\mu| \gtrsim 110 \mathrm{GeV}$ with $\mu<0$, since elsewhere the chargino is heavier than $91 \mathrm{GeV}$. In this $|\mu|$ range, the decay $h \rightarrow \chi_{1}^{0} \chi_{1}^{0}$ is kinematically allowed, but the branching ratio is very small, less than $0.5 \%$. This is due to the fact that in this area $\chi_{0}^{1}$ is a pure bino state and its couplings to the $h$ boson are strongly suppressed. What makes the $h \rightarrow \gamma \gamma$ branching ratio drop by almost a factor two compared to the previous case is first, the smaller value of $M_{h}$ [the decay width grows with the third power of the Higgs mass] and then because of the contribution of the chargino loops to the $h \rightarrow \gamma \gamma$ decay, which interfere destructively for $\mu<0$ with the dominant contribution due $W$ boson loops [the reduction is nevertheless very mild, at most $15 \%$ in this case]. 


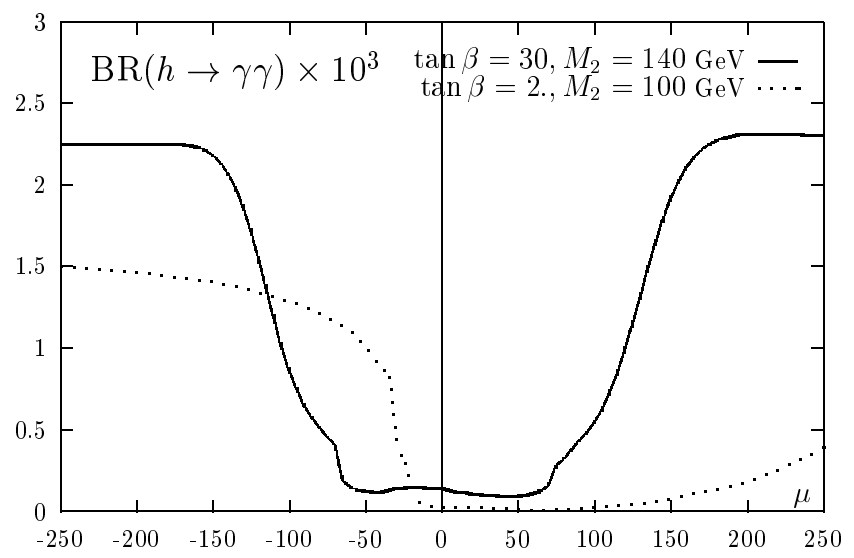

Fig. 1. Branching ratios in units of $10^{-3}$ for the decays $h \rightarrow \gamma \gamma$ as a function of $\mu$ for $\tan \beta=2(30)$ and $M_{2}=100(140) \mathrm{GeV}$.

If the constraint on the unification of the gaugino masses at the GUT scale is relaxed, there is practically no lower bound on the LSP mass. Indeed, for relatively large $\mu$ values, the lightest chargino $\chi_{1}^{+}$and the next-to-lightest neutralino $\chi_{2}^{0}$ are wino-like with a mass $\sim M_{2}$ while the lightest neutralino is bino-like with a mass $\sim M_{1}$; since $M_{1}$ is a free parameter, it can be as small as possible leading to a possibly very light LSP. The decay $h \rightarrow \chi_{0}^{1} \chi_{0}^{1}$ will then have more room to occur.

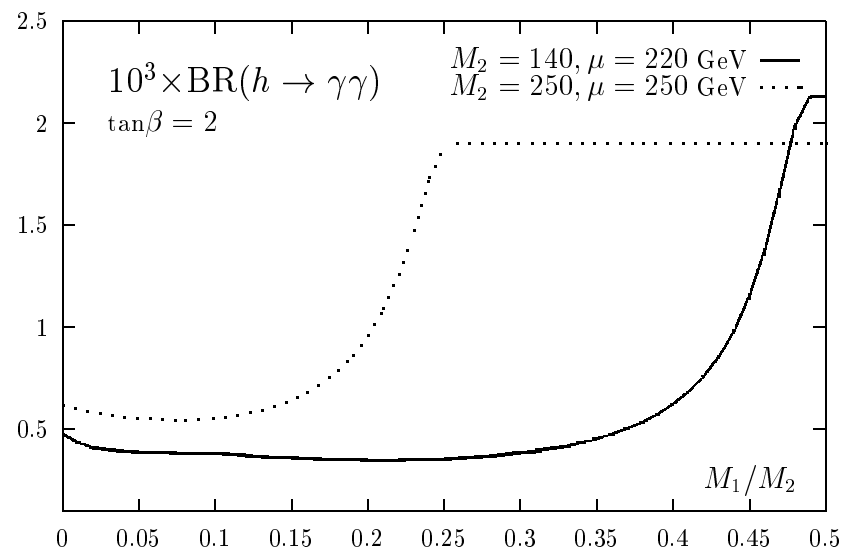

Fig. 2. Branching ratios for the decays $h \rightarrow \gamma \gamma$ in units of $10^{-3}$ as a function of $M_{1} / M_{2}$ for $\tan \beta=2$ and two sets of $M_{2}, \mu$ values.

The branching ratio for the decay $h \rightarrow \chi_{1}^{0} \chi_{1}^{0}$ can be rather large thus suppressing the $\gamma \gamma$ branching ratio. This is exemplified in Fig. 2, where $\operatorname{BR}(h \rightarrow \gamma \gamma)$ is shown as a function of the ratio $M_{1} / M_{2}$ for $\tan \beta=2$ and for two sets of $M_{2}$ and $\mu$ values; $M_{2}=140 \mathrm{GeV}, \mu=220 \mathrm{GeV}$ leading to $m_{\chi_{1}^{+}} \simeq 96 \mathrm{GeV}$, and $M_{2}=\mu=250$ $\mathrm{GeV}$ leading to $m_{\chi_{1}^{+}} \simeq 175 \mathrm{GeV}$; the remaining inputs are as in the previous figure. 
In the first scenario, when the LSP is very light $\mathrm{BR}(h \rightarrow \gamma \gamma)$ drops to the level of $5.10^{-4}$, a strong reduction compared to the expected rate $\sim 2.10^{-3}$. With increasing $M_{1} / M_{2}$ and hence with increasing LSP mass, it stays almost constant until the $2 m_{\chi_{1}^{0}}$ threshold is reached for $M_{1} \sim M_{2} / 2$ and the rate recovers its standard value. In the second scenario, $\operatorname{BR}(h \rightarrow \gamma \gamma)$ starts at the same level as previously, but increases more rapidly and reaches approximately the standard value for $M_{1} \sim M_{2} / 4$ which corresponds to the kinematical limit for the decay $h \rightarrow \chi_{1}^{0} \chi_{1}^{0}$. When the LSP decay is shut, the difference between the $\gamma \gamma$ branching ratios in the two scenarios is due to a constructively interfering chargino loop contribution [the sign of the $\chi_{1}^{ \pm}$ contribution goes with the sign of $\mu]$ in the case where $m_{\chi_{1}^{+}} \simeq 96 \mathrm{GeV}$ and which enhances the $\gamma \gamma$ decay width by $20 \%$ or so. This picture is expected not to be altered significantly for larger values of $\tan \beta$ if the $h$ boson is in the decoupling regime as discussed previously [in fact for large $\tan \beta$ values and for some moderate values of the parameters $M_{2}$ and $\mu$, even the decays into the lightest and the next to lightest neutralinos is possible].

Another kinematically still possible SUSY mode for the lightest $h$ boson is the decay into sneutrinos. Indeed the experimental lower bound on the $\tilde{\nu}$ masses is still rather low, $m_{\tilde{\nu}} \gtrsim 45 \mathrm{GeV}$, leaving some room for the decay $h \rightarrow \tilde{\nu} \tilde{\nu}$ to occur. However, because of $\mathrm{SU}(2)_{\mathrm{L}}$ invariance, the sneutrino and the left-handed charged slepton masses are related and one should avoid being into conflict with the stronger experimental bound $m_{\tilde{l}_{L}} \gtrsim 70 \mathrm{GeV}$. However, even in this case one can obtain a rather light sneutrino since a splitting between the $\tilde{\nu}$ and $\tilde{l}_{L}$ masses can be generated by the D-terms. Indeed, denoting the common scalar mass by $\tilde{m}$, one has:

$$
m_{\tilde{\nu}}^{2} \simeq \tilde{m}^{2}+0.50 M_{Z}^{2} \cos 2 \beta, m_{\tilde{l}_{L}}^{2} \simeq \tilde{m}^{2}-0.27 M_{Z}^{2} \cos 2 \beta
$$

For small values of $\tilde{m}$, the slepton masses are governed by the $\mathrm{D}$-terms, and for large values of $\tan \beta, \cos 2 \beta \rightarrow-1$ and the $\mathrm{D}$-terms become maximal. Since they tend to increase $m_{\tilde{l}_{L}}$ and decrease $m_{\tilde{\nu}}$, relatively low masses for sneutrinos can be kept while still having rather heavy left-handed ${ }^{d}$ sleptons [note however, that $\tilde{\nu}$ should not be lighter than the lightest neutralino which is expected to be the LSP].

In the decoupling limit, the $h$ boson coupling to sneutrinos is also proportional to $\cos 2 \beta$, and for large $\tan \beta$ values it becomes maximal. And since it is a "gauge" coupling, it is much larger than the $h b \bar{b}$ Yukawa coupling, and the decay $h \rightarrow \tilde{\nu} \tilde{\nu}$ is always largely dominating once it is kinematically allowed. The partial width for the decay, summing over the three sneutrinos, is given by

$$
\Gamma(h \rightarrow \tilde{\nu} \tilde{\nu})=\frac{3 G_{F} M_{Z}^{4}}{8 \sqrt{2} \pi M_{h}} \beta_{\tilde{\nu}}, \quad \beta_{\tilde{\nu}}=\left[1-\frac{4 m_{\tilde{\nu}}^{2}}{M_{h}^{2}}\right]^{1 / 2}
$$

Modulo the velocity factor $\beta_{\tilde{\nu}}$, the partial width is larger than the otherwise dominant $b \bar{b}$ decay width by a huge factor: $M_{Z}^{4} /\left(2 m_{b}^{2} M_{h}^{2}\right) \sim 230$ for $M_{h}=130 \mathrm{GeV}$.

\footnotetext{
${ }^{d}$ The D-terms for right-handed charged sleptons are approximately the same as for the left-handed ones and tend also to decrease the mass. However, in GUT scenarii such as mSUGRA, the $\tilde{l}_{R}$ tends to be lighter than the sneutrinos for reasonable values of the gaugino mass $m_{1 / 2}$. In this case, the decay $h \rightarrow \tilde{\nu} \tilde{\nu}$ is forbidden because of the experimental bound $m_{\tilde{l}_{R}} \gtrsim 70 \mathrm{GeV}$.
} 
Thus, if the $h \rightarrow \tilde{\nu} \tilde{\nu}$ decay mode is allowed, all the branching ratios for the other decay channels including the $h \rightarrow \gamma \gamma$ mode, will be suppressed by two orders of magnitude. Since the sneutrinos will decay invisibly in this mass range $\left[m_{\tilde{\nu}}<m_{\chi_{1}^{ \pm}}\right.$ and the only possible channel is the invisible mode $\left.\tilde{\nu} \rightarrow \nu \chi_{1}^{0}\right]$, the $h$ boson would be then also very difficult to detect at the LHCe.

\section{H/A decays into SUSY particles}

If the $\mathrm{CP}$-even and the $\mathrm{CP}$-odd Higgs bosons $H$ and $A$ are heavy, $M_{H, A} \gtrsim 300$ $\mathrm{GeV}$, at least the decays into the lightest neutralinos and possibly charginos should be kinematically allowed. For moderate $\tan \beta$ values, the couplings to $b \bar{b}$ and $\tau^{+} \tau^{-}$ pairs [which together with $t \bar{t}$ states account for the total width in the absence of SUSY modes] are not strongly enhanced, these decays might be dominant and suppress drastically the branching ratios for the $H / A \rightarrow \tau^{+} \tau^{-}$signals. The partial widths for the decays of the particle $\Phi=H, A$ into $\chi_{i} \chi_{j}$ states are given by 4

$\Gamma=\frac{G_{F} M_{W}^{2} M_{\Phi} \lambda_{i j}^{1 / 2}}{2 \sqrt{2} \pi\left(1+\delta_{i j}\right)}\left[\left(F_{i j \Phi}^{2}+F_{j i \Phi}^{2}\right)\left(1-\frac{m_{\chi_{i}}^{2}}{M_{\Phi}^{2}}-\frac{m_{\chi_{j}}^{2}}{M_{\Phi}^{2}}\right)-4 \eta_{\Phi} \epsilon_{i} \epsilon_{j} F_{i j \Phi} F_{j i \Phi} \frac{m_{\chi_{i}} m_{\chi_{j}}}{M_{\Phi}^{2}}\right]$

where $\eta_{H}=+1, \eta_{A}=-1$ and $\delta_{i j}=0$ unless the final state consists of two identical (Majorana) neutralinos in which case $\delta_{i i}=1_{i} \epsilon_{i}= \pm 1$ stands for the sign of the $i$ 'th eigenvalue of the neutralino mass matrix 15 while $\epsilon_{i}=1$ for charginos; $\lambda_{i j}=\left(1-m_{\chi_{i}}^{2} / M_{\Phi}^{2}-m_{\chi_{j}}^{2} / M_{\Phi}^{2}\right)^{2}-4 m_{\chi_{i}}^{2} m_{\chi_{j}}^{2} / M_{\Phi}^{4}$. The coefficients $F_{i j \Phi}$ are related to the elements of the matrices $15 U, V$ and $Z$ for charginos and neutralinos; in the decoupling limit they are the same for $H$ and $A$ and read

$$
\begin{array}{ll}
\Phi \rightarrow \chi_{i}^{0} \chi_{j}^{0} \quad: \quad F_{i j \Phi}=\frac{1}{2}\left(Z_{j 2}-\tan \theta_{W} Z_{j 1}\right)\left(\sin \beta Z_{i 3}-\cos \beta Z_{i 4}\right)+i \leftrightarrow j \\
\Phi \rightarrow \chi_{i}^{+} \chi_{j}^{-} \quad: \quad F_{i j \Phi}=\frac{1}{\sqrt{2}}\left[\sin \beta V_{i 1} U_{j 2}+\cos \beta V_{i 2} U_{j 1}\right]
\end{array}
$$

In Fig. 3, the branching fractions $\operatorname{BR}\left(H / A \rightarrow \tau^{+} \tau^{-}\right)$are plotted as a function of the $H / A$ masses for three values $\tan \beta=5,10$ and 30 . The choice $M_{2}=-\mu=200$ $\mathrm{GeV}$ has been made leading to $m_{\chi_{1}^{0}} \sim 90 \mathrm{GeV}$ and $m_{\chi_{1}^{+}} \sim 160 \mathrm{GeV}$ [with a small variation with $\tan \beta]$. The branching ratios for $H$ and $A$ decays are almost the same except for small values of $\tan \beta$ and relatively small Higgs masses: in this case, the decoupling limit is not yet reached and additional [and different] decay modes occur for the $H$ and $A$ bosons as discussed previously. For $\tan \beta=5$ the $H / A$ couplings to down-type fermions are not very strongly enhanced and the decays into charginos and neutralinos have large branching ratios: they decrease $\operatorname{BR}\left(H / A \rightarrow \tau^{+} \tau^{-}\right)$from the standard $\sim 10 \%$ value for small Higgs masses [where only a few SUSY channels are open and some are suppressed by phase space] to less than $0.4 \%$ for very heavy Higgs boson masses $M_{H, A} \sim 1 \mathrm{TeV}$ [here most of the neutralino/chargino channels are open and they are not suppressed by phase space], thus a reduction by more than a factor of 20 compared to the branching ratio without the SUSY decays. For $\tan \beta=10$, the couplings to $b$-quarks and $\tau$-leptons are more enhanced and

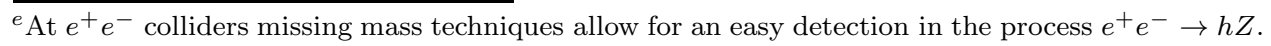




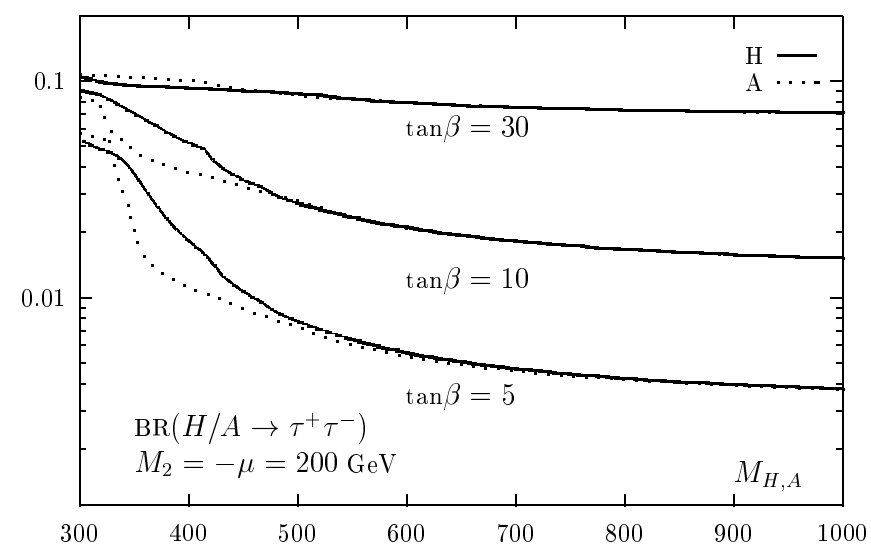

Fig. 3. Branching ratios for the decays $H / A \rightarrow \tau^{+} \tau^{-}$as a function of $M_{H, A}$ for $\tan \beta=5,10$ and 30 and for the values $M_{2}=-\mu=200 \mathrm{GeV}$.

$\operatorname{BR}\left(H / A \rightarrow \tau^{+} \tau^{-}\right)$are larger by slightly more than a factor of two compared to the previous case. For even larger values of $\tan \beta, \tan \beta=30$, the decays into charginos and neutralinos are not dominating anymore, and the branching ratios for the $H / A$ decays into tau pairs are suppressed only slightly, less than a factor of two.

In the preceding discussion, the decays of $H$ and $A$ into sfermions were assumed to be shut. However, at least the decays into the lightest stops can be kinematically allowed, and strongly enhanced $f$ Indeed, the current stop eigenstates, $\tilde{t}_{L}$ and $\tilde{t}_{R}$, mix to give the mass eigenstates $\tilde{t}_{1}$ and $\tilde{t}_{2}$; the mixing angle $\theta_{\tilde{t}}$ is proportional to $A_{t}-\mu / \tan \beta$, and can be very large, leading to a $\tilde{t}_{1}$ much lighter than the $t$-quark and all other scalar quarks. In addition the couplings of the top squarks to the Higgs boson $H$ in the decoupling limit read

$g_{H \tilde{t}_{1} \tilde{t}_{1}}=\sin 2 \beta\left[\frac{1}{2} \cos ^{2} \theta_{\tilde{t}}-\frac{2}{3} s_{W}^{2} \cos 2 \theta_{\tilde{t}}\right]-\frac{m_{t}^{2}}{M_{Z}^{2}} \frac{1}{\tan \beta}+\frac{1}{2} \sin 2 \theta_{\tilde{t}} \frac{m_{t}}{M_{Z}^{2}}\left(\frac{A_{t}}{\tan \beta}+\mu\right)$

For large values of $A_{t}$ or $\mu$, which incidentally make $\theta_{\tilde{t}}$ maximal, $\left|\sin 2 \theta_{\tilde{t}}\right| \simeq 1$, the last components can strongly enhance the $g_{H \tilde{t} \tilde{t}}$ couplings and make them larger than the top quark coupling of the $H$ boson, $g_{H t t} \propto m_{t} / M_{Z}$. The pseudoscalar $A$ couples only to $\tilde{t}_{1} \tilde{t}_{2}$ pairs because of $\mathrm{CP}$-invariance, the coupling is given by:

$$
g_{A \tilde{t}_{1} \tilde{t}_{2}}=\frac{1}{2} \frac{m_{t}}{M_{Z}^{2}}\left(A_{t} / \tan \beta-\mu\right)
$$

In the maximal mixing case, $\left|\sin 2 \theta_{\tilde{t}}\right| \simeq 1$, this is also the main component of the $H$ boson coupling to $\tilde{t}_{1} \tilde{t}_{2}$ pairs except that the sign of $\mu$ is reversed.

The partial decay widths of the $H, A$ bosons into top squarks are given by

$$
\Gamma\left(\Phi \rightarrow \tilde{t}_{i} \tilde{t}_{j}\right)=\frac{3 G_{F}}{2 \sqrt{2} \pi M_{\Phi}} \lambda_{\tilde{f}_{i} \tilde{f}_{j}}^{1 / 2} g_{\Phi \tilde{t}_{i} \tilde{t}_{j}}^{2}
$$

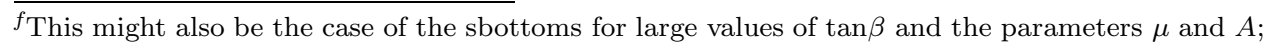
however this will not be discussed here and we will assume that the mixing is zero in this sector. 
For the $H$ boson, the partial width, up to mixing angle factors is proportional to $G_{F} m_{t}^{4} /\left(M_{H} \tan ^{2} \beta\right)$ or/and $G_{F} m_{t}^{2}\left(\mu-A_{t} / \tan \beta\right)^{2} / M_{H}$; for small $\tan \beta$ values and not too large $M_{H}$ and for intermediate $\tan \beta$ values and for large $\mu$ and $A_{t}$, the width for the decays $H \rightarrow \tilde{t} \tilde{t}$ can be very large and can compete with, and even dominate over, the other [standard and SUSY] decay channels. The branching ratios for the $H$ decays into $\tau$ pairs would be then further suppressed.

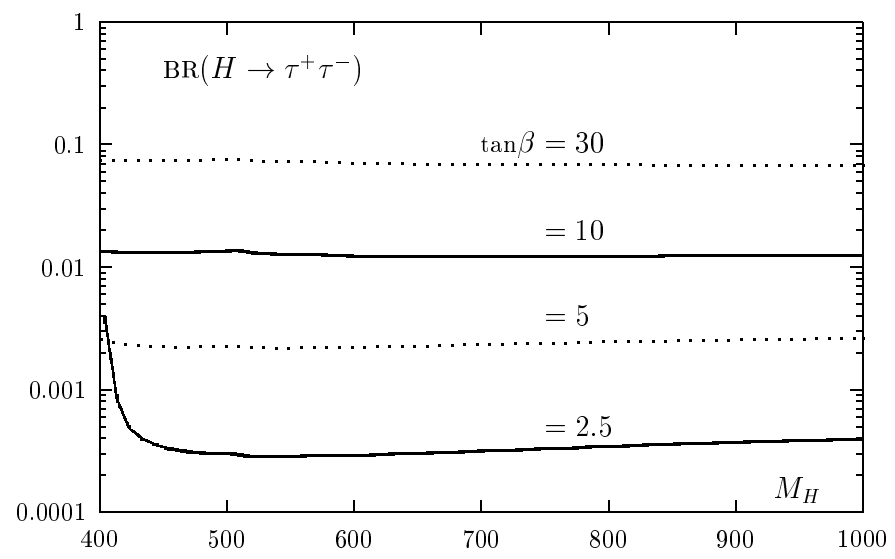

Fig. 4. Branching fractions for $H \rightarrow \tau^{+} \tau^{-}$as a function of $M_{H}$ for $\tan \beta=2.5,5,10,30, m_{\tilde{t}_{1}} \simeq 200$ $\mathrm{GeV}, M_{2}=\mu=m_{\tilde{f}} / 2=250 \mathrm{GeV}$ and $A_{t}=1.5 \mathrm{TeV}$.

This is illustrated in Fig. 4, where $\operatorname{BR}\left(H \rightarrow \tau^{+} \tau^{-}\right)$is shown as a function of $M_{H}$ for $\tan \beta=2.5,5,10,30$ and $m_{\tilde{t}_{1}} \simeq 200 \mathrm{GeV}$ [for $\tan \beta=2.5$ this is achieved by setting $m_{\tilde{f}_{L}}=m_{\tilde{f}_{R}}=500 \mathrm{GeV}$ and $\left.A_{t}=1.5 \mathrm{TeV}\right] ; M_{2}=\mu=250 \mathrm{GeV}$. $\operatorname{BR}\left(H \rightarrow \tilde{t}_{1} \tilde{t}_{1}\right)$ decreases with increasing $\tan \beta$ values and increasing $M_{H}$, but it is still at the level of $\sim 50 \%$ for $\tan \beta=5$ and $M_{H}=1 \mathrm{TeV}$. For $\tan \beta=30$, the channel $H \rightarrow \tilde{t}_{1} \tilde{t}_{2}$ opens up for $M_{H} \sim 900 \mathrm{GeV}$; however for this large $\tan \beta$ value, the branching ratio barely exceeds the level of $20 \%$ in contrast to lower $\tan \beta$ values where it can reach almost unity for small $M_{H}$. For larger $M_{H}$, the decays into charginos and neutralinos become more important and will dominate; so $\operatorname{BR}\left(H \rightarrow \tau^{+} \tau^{-}\right)$is reduced anyway.

For the $A$ boson the only important decay into sfermions is $A \rightarrow \tilde{t}_{1} \tilde{t}_{2}$ [and maybe $\tilde{b}_{1} \tilde{b}_{2}$ for $\left.\tan \beta \gg 1\right]$. Thus both stops must be light for the decay to be allowed by kinematics. This happens only in a small area of the parameter space, unless all squarks are relatively light. For instance, in the scenario above, $m_{\tilde{t}_{2}} \sim 700 \mathrm{GeV}$ and the decays $A, H \rightarrow \tilde{t}_{1} \tilde{t}_{2}$ occur only for masses close to $1 \mathrm{TeV}$. Note that the decay widths of the $H$ bosons into the light fermion partners are proportional to $G_{F} M_{W}^{4} \sin ^{2} 2 \beta / M_{H}$ for $M_{H} \gg m_{\tilde{f}}$. They are thus suppressed by the heavy $H$ mass and cannot compete with the decays into fermions $[t, b, \tau$ and possibly $\chi$ states $]$ for which the widths grow as $M_{H}$. The pseudoscalar $A$ boson cannot decay into the partners of light fermions, if the fermion mass is neglected. 


\section{Conclusions}

I have discussed the SUSY decay modes of the neutral Higgs bosons in the MSSM. Decays of the $h$ boson into invisible neutralinos [and also sneutrinos] are still possible, especially if the gaugino mass unification constraint at the GUT scale is relaxed, and might be dominant when they occur, hence reducing the $h \rightarrow \gamma \gamma$ branching ratio significantly. Decays of the $H$ and $A$ bosons into chargino and neutralino pairs, and decays of the $H$ boson into stops, are also important in large areas of the MSSM parameter space, and can suppress strongly the branching ratios for the $\tau^{+} \tau^{-}$discovery mode. These decay modes should not be overlooked, as they might jeopardize the search of the MSSM Higgs bosons at the LHC.

\section{Acknowledgements}

The work presented here has been initiated after stimulating discussions during the GDR-Supersymétrie workshop.

\section{References}

1. For a review on the Higgs sector of the MSSM, see J.F. Gunion, H.E. Haber, G.L. Kane and S. Dawson, "The Higgs Hunter's Guide", Addison-Wesley, Reading 1990.

2. ATLAS Collaboration, Technical Proposal, Report CERN-LHCC 94-43; CMS Collaboration, Technical Proposal, Report CERN-LHCC 94-38.

3. For as recent review of Higgs boson searches at the LHC, see e.g.: J.F. Gunion et al., hep-ph/9602238. Proceedings of the Snowmass 96 Workshop; See also: M. Spira, Habilitation thesis, hep-ph/9705337.

4. D. Froidevaux et al., ATLAS internal note PHYS-no-74 (1995).

5. For a recent collection of experimental constraints, see the LEPC Jamboree, March 31, 1998 and A. Djouadi et al., hep-ph/9901246.

6. M. Carena, M. Quiros and C.E.M. Wagner, Nucl. Phys. B461 (1996) 407; H. Haber, R. Hempfling and A. Hoang, Z. Phys. C75 (1997) 539; S. Heinemeyer, W. Hollik and G. Weiglein, hep-ph/9803277.

7. H. Georgi et al., Phys. Rev. Lett. 40 (1978) 692. A. Djouadi, M. Spira and P.M. Zerwas, Phys. Lett. B264 (1991) 440; S. Dawson, Nucl. Phys. B359 (1991) 283; M. Spira, A. Djouadi, D. Graudenz and P.M. Zerwas, Nucl. Phys. B453 (1995) 17; S. Dawson, A. Djouadi and M. Spira, Phys. Rev. Lett. 77 (1996) 16.

8. S.L. Glashow, D.V. Nanopoulos and A. Yildiz, Phys. Rev. D18 (1978) 1724; Z. Kunszt, Z. Trocsanyi and W.J. Stirling, Phys. Lett. B271 (1991) 247.

9. Z. Kunszt, Nucl. Phys. B247 (1984) 339; J.F. Gunion, Phys. Lett. B253 (1991) 269; W. Marciano and F. Paige, Phys. Rev. Lett. 66 (1991) 2433.

10. A. Djouadi, Phys. Lett. B435 (1998) 101.

11. A. Djouadi, J.L. Kneur and G. Moultaka, Phys. Rev. Lett. 80 (1998) 1830.

12. M. Carena, P.M. Zerwas et al., Yellow Report CERN-96-01 and hep-ph/9602250.

13. A. Djouadi, J. Kalinowski and M. Spira, Comput. Phys. Commun. 108 (1998) 56.

14. J.F. Gunion and H.E. Haber, B307 (1988) 445; A. Djouadi, J. Kalinowski and P.M. Zerwas, Z. Phys. C57 (1993) 569; H. Baer et al., Phys. Rev. D47 (1993) 1062; A. Djouadi, P. Janot, J.Kalinowski and P.M. Zerwas, Phys. Lett. B376 (1996) 220; A. Djouadi, J. Kalinowski, P. Ohmann and P.M. Zerwas, Z. Phys. C74 (1997) 93.

15. H. E. Haber and G. Kane, Phys. Rep. 117 (1985) 75. 